\title{
Phenotypic characterization of the novel, non-hotspot oncogenic KRAS mutants E31D and E63K
}

\author{
ARLOU KRISTINA J. ANGELES ${ }^{1,4}$, RYAN TIMOTHY D. YU ${ }^{1}$, \\ EVA MARIA CUTIONGCO-DE LA PAZ ${ }^{2,3}$ and REYNALDO L. GARCIA ${ }^{1,3}$
}

\begin{abstract}
${ }^{1}$ Disease Molecular Biology and Epigenetics Laboratory, National Institute of Molecular Biology and Biotechnology, National Science Complex, University of the Philippines Diliman, Quezon City 1101; ${ }^{2}$ Institute of Human Genetics, National Institutes of Health, University of the Philippines Manila, Manila 1000; ${ }^{3}$ Philippine Genome Center, University of the Philippines System, Quezon City 1101, Philippines
\end{abstract}

Received October 20, 2018; Accepted April 2, 2019

DOI: $10.3892 / \mathrm{ol} .2019 .10325$

\begin{abstract}
KRAS proto-oncogene, GTPase (KRAS) functions as a molecular switch at the apex of multiple signaling pathways controlling cell proliferation, differentiation, migration, and survival. Canonical KRAS mutants, such as those in codons 12 and 13, produce constitutively active oncoproteins that short-circuit epidermal growth factor receptor (EGFR)-initiated signaling, resulting in dysregulated downstream effectors associated with cellular transformation. Therefore, anti-EGFR therapy provides little to no clinical benefit to patients with activating KRAS mutations. Current genotyping procedures based on canonical mutation detection only account for $\sim 40 \%$ of non-responders, highlighting the need to identify additional predictive biomarkers. In the present study, two novel non-hotspot KRAS mutations were functionally characterized in vitro: KRAS E31D was identified from a genetic screen of colorectal cancer specimens at the UP-National Institutes of Health. KRAS E63K is curated in the Catalogue of Somatic Mutations in Cancer database. Similar to the canonical mutants KRAS G12D and KRAS G13D, NIH3T3 cells overexpressing KRAS E31D and KRAS E63K showed altered morphology and were characteristically smaller, rounder, and highly refractile compared with their non-transformed counterparts. Filamentous actin staining also indicated cytoplasmic shrinkage, membrane ruffling, and
\end{abstract}

Correspondence to: Professor Reynaldo L. Garcia, Disease Molecular Biology and Epigenetics Laboratory, National Institute of Molecular Biology and Biotechnology, National Science Complex, University of the Philippines Diliman, Regidor Street, Quezon City 1101, Philippines

E-mail: reygarcia@mbb.upd.edu.ph

Present address: ${ }^{4}$ Division of Cancer Genome Research, German Cancer Research Center and National Center for Tumor Diseases, Im Neuenheimer Feld 460, Heidelberg, D-69120, Germany

Key words: KRAS proto-oncogene, GTPase, colorectal cancer, oncogene, carcinogenesis, epidermal growth factor receptor pathway formation of pseudopod protrusions. Further, they displayed higher proliferative rates and higher migratory rates in scratch wound assays compared with negative controls. These empirical findings suggest the activating impact of the novel KRAS mutations, which may contribute to resistance to anti-EGFR therapy. Complementary studies to elucidate the molecular mechanisms underlying the transforming effect of the rare mutants are required. In parallel, their oncogenic capacity in vivo should also be investigated.

\section{Introduction}

In the clinical setting, KRAS Proto-Oncogene, GTPase (KRAS) genotyping is a powerful predictive tool used to stratify patients with colorectal cancer (CRC) for anti-epidermal growth factor receptor (EGFR) therapy (1). KRAS is a molecular switch protein at the apex of multiple signaling cascades that control diverse biological processes, including cell proliferation, differentiation and apoptosis (2). Activating KRAS mutations, known to occur in $35-40 \%$ of colorectal tumors, result in the expression of active oncoproteins that continuously transmit positive growth signals to downstream effectors (3). As KRAS functions downstream of EGFR, blocking the receptor provides little therapeutic benefit to patients with activating KRAS mutations. KRAS testing is therefore, imperative prior to anti-EGFR indication to avoid administration of ineffective, potentially toxic, and highly expensive therapy $(4,5)$.

Activating KRAS mutations in CRC are single point mutations found mostly in codons 12 or 13 of exon 2, and codon 61 of exon 3 (6). These missense mutations produce constitutively active protein products capable of initiating cellular transformation in relevant cell line models including primary mouse embryonic fibroblasts (MEFs) (7), murine NIH3T3 (8) and rat Rat-1 fibroblasts (9). Although the morphological, functional, and molecular features conferred by these mutations have already been extensively studied, current KRAS genotyping guidelines limited to testing only for these canonical mutants could present possible complications $(10,11)$. Firstly, occurrence of canonical KRAS mutations only accounts for $30-40 \%$ of anti-EGFR non-responders (4). Hence, additional 
predictive markers, such as genetic lesions downstream of KRAS, including B-Raf proto-oncogene, serine/threonine kinase, phosphatidylinositol-4,5-bisphosphate 3-kinase catalytic subunit $\alpha$, phosphatase and tensin homolog or in the NRAS proto-oncogene GTPase isoform, are currently being investigated as potential biomarkers in association with anti-EGFR therapy, for patients with the wild-type KRAS allele $(5,12,13)$. Another problem lies with the fact that the bulk of epidemiological data used to establish the predominance of the canonical KRAS mutations in CRC were collected from western countries and other developed nations (14). In addition, based on the varying epidemiological distribution of mutations reported in different countries such as the U.K. (27.4\%) (14), Switzerland (37\%) (15), Jordan (44\%) (16) and China (42.2\%) (17), KRAS mutation frequency and spectrum in CRC are most likely dependent on the population under study $(14,18)$. In light of this paradigm, investigations on the functional consequence of rare and novel KRAS mutations across populations are crucial in improving CRC diagnostics, prognostics, and therapeutics.

Between 2003 and 2010, a retrospective survey of exon 2 KRAS mutations in 153 histopathologically verified malignant colorectal tissue samples at the University of the Philippines Manila-National Institutes of Health revealed novel mutations in addition to the canonical mutations in codons 12 and 13 (unpublished data: Cutiongco-Dela Paz et al). One such mutation was observed in KRAS exon 2, codon 31 (NM_004985.3: c.93A $>\mathrm{T})$. The significance of this mutation is currently unknown. This missense mutation changes the amino acid at position 31 from glutamate to aspartate (p.31E>D; E31D). Another putative activating KRAS mutation detected in CRC tissues was reported for codon 63 in exon 2 (19). This mutation (NM_004985.3: c.187G>A) changes a glutamic acid residue into lysine (p.63E $>\mathrm{K}$; E63K), strongly hinting at probable functional alterations in the wild-type protein due to charge alteration at a position critical in stabilizing the guanosine triphosphate (GTP) hydrolysis reaction (20). Similar to the novel mutation found in codon 31 , functional characterization studies of the codon 63 mutant, to the best of our knowledge, have yet to be reported in the literature. In the present study, the functional characterization of the E31D and E63K mutations in KRAS were reported. Their effects on proliferative rates, invasiveness, cytoskeletal organization and general morphology were investigated.

\section{Materials and methods}

Cloning and site-directed mutagenesis of wild-type and KRAS mutant variants. The wild-type KRAS isoform b precursor (NM_004985.3) was re-amplified from a previous clone in pGem ${ }^{\circledR}$-T Easy (Promega Corporation, Madison, WI, USA) available in the laboratory, using wild-type primers appended with restriction sites for HindIII and Not I at the $5^{\prime}$ and $3^{\prime}$ ends, respectively. The primers used to generate all KRAS variants used in this study are as follows: KRAS, F 5'-GCCGTCCTGAAGCTTCGCCGGATGACTGAATATAAA CTTG-3', R 5'-GTCGTGCCGCGGCCGCGTGCCGTTACA TAATTACACACTTTG-3'; KRAS E31D, R 5'-GTTGGA TCATAA*TCGTCCAC-3', F 5'-GTGGACGAT*TATGAT CCAAC-3'; KRAS E63K, R 5'-GCACTGTACTT*CTCTTG
ACC-3', F 5'-GGTCAAGAGA*AGTACAGTGC-3'; KRAS G12D, F 5'-GCCGTCCTGAAGCTTCGCCGGATGACT GAATAT; AAACTTGTGGTAGTTGGAGCTGA*TGGCGT AGG-3'; KRAS G13D, F 5'-GCCGTCCTGAAGCTTCGC CGGATGACTGAATAT; AAACTTGTGGTAGTTGGAGCT GGTGA*CGTAGG-3'.

Site-directed mutagenesis by overlap extension PCR was used to generate the mutants. The general PCR components and conditions used in this study are as follows. Each reaction mixture contained a final concentration of $1 \mathrm{X}$ PCR buffer (Titanium ${ }^{\circledR}$ Taq PCR buffer, Clontech Laboratories, Inc., Mountain View, CA, USA), $0.125 \mathrm{mM}$ of each deoxynucleoside triphosphate (iNtRON Biotechnology, Inc., Sangdaewon-Dong, Jungwon-Seongnam, South Korea), $2 \mathrm{mM}$ each of the appropriate forward and reverse primers, 1X Taq polymerase (Titanium ${ }^{\circledR}$ Taq polymerase, Clontech Laboratories), and $50 \mathrm{ng}$ of the cloned pTargeT ${ }^{\mathrm{TM}}$-KRAS WT template. The template DNA was initially denatured at $94^{\circ} \mathrm{C}$ for $5 \mathrm{~min}$. Twenty cycles of denaturation at $94^{\circ} \mathrm{C}$ for $5 \mathrm{~min}$, annealing at $55^{\circ} \mathrm{C}$ for $30 \mathrm{sec}$, and extension at $72^{\circ} \mathrm{C}$ for $1 \mathrm{~min}$ were then performed using the $\mathrm{C} 1000 \mathrm{Touch}^{\mathrm{TM}}$ Thermal Cycler (Bio-Rad Laboratories, Inc., Hercules, CA, USA).

Two fragments of the same mutant were then fused using overlap extension-PCR, where $25 \mathrm{ng}$ of each fragment was used as a template with KRAS-F and KRAS-R as primers. The same PCR conditions stated above were used with the additions of a final extension step at $72^{\circ} \mathrm{C}$ for $10 \mathrm{~min}$ after 20 cycles of amplification to ensure the addition of A-overhangs for TA-cloning into the pTargeT $^{\mathrm{TM}}$ mammalian expression vector (Promega Corporation). Amplified PCR products were cloned directly into pTarge ${ }^{\mathrm{TM}}$ and verified error-free and in the correct orientation by Sanger sequencing.

Cell culture and transfection of NIH3T3 cells. NIH3T3 cells were purchased from the American Type Culture Collection (ATCC; Manassas, Virginia, USA; cat. no. CRL-1658). The cells were maintained in Dulbecco's modified Eagle's medium (DMEM; Gibco; Thermo Fisher Scientific, Inc., Waltham, MA, USA) supplemented with $10 \%$ bovine calf serum (BCS; Gibco; Thermo Fisher Scientific, Inc.), $3 \mathrm{mg} / \mathrm{ml}$ sodium bicarbonate $\left(\mathrm{NaHCO}_{3}\right), 100 \mathrm{U} / \mathrm{ml}$ penicillin/streptomycin, and incubated in a humidified atmosphere containing $5 \% \mathrm{CO}_{2}$ at $37^{\circ} \mathrm{C}$. Cells were seeded onto 12-well polypropylene culture plates. At $80-90 \%$ confluency, the cells in each well were transfected with $2 \mu \mathrm{g}_{\text {pTarge }}{ }^{\mathrm{TM}}$ construct, using $4 \mu \mathrm{l} \mathrm{Lipofectamine}^{\mathrm{TM}} 2000$ (Invitrogen; Thermo Fisher Scientific, Inc.). A mammalian expression vector containing a green fluorescent protein gene reporter (pmR-ZsGreen1; Clontech Laboratories) was also transfected vis-à-vis the KRAS expression constructs to approximate transfection efficiency. For particular experiments, each pTargeT ${ }^{\mathrm{TM}}$ construct was co-transfected with pmR-ZsGreen1 in a 1:7 vector ratio (pmiR-ZsGreen1: pTargeT) to fluorescently track cells that were most likely transfected and are overexpressing the KRAS variant. Transfection efficiency was assessed by fluorescence microscopy between 24 and $72 \mathrm{~h}$ post-transfection, and the cells were observed or harvested for subsequent morphological, functional and molecular characterization experiments. Routinely and periodically, all constructs were also tested for ability to express the transcript and/or protein via RT-PCR and western blot, respectively. 
Morphological characterization. Morphological appearance, including size, refringency, presence of filopodia, presence of lamellipodia, and depolarization of transfected fibroblasts was examined under an inverted brightfield microscope at 100x magnification $48 \mathrm{~h}$ post-transfection. To quantitatively compare the transforming effect on cellular morphology by the different forms of KRAS, percentage of cells exhibiting transformed characteristics was determined for each transfection setup. Each transfected well was imaged in three random fields of view. Using the Fiji opensource platform for biological image analysis (21), fibroblasts with aberrant morphology were counted for each documented field. A total cell count per view was also performed. The mean percentage of morphologically transformed cells was then computed for all fields of view. The values were then statistically compared among all transfectants.

Monitoring changes in actin cytoskeletal architecture. NIH3T3 cells were seeded into an 8-well glass chamber slide (EMD Millipore, Burlington, MA, USA), and transfected the next day as described previously. Forty-eight hours post-transfection, the cells were fixed, permeabilized, and stained with a phalloidin fluorescent conjugate. All steps were performed at room temperature. Briefly, cells were washed twice with 1X PBS, and fixed for 20 min using $4 \%$ paraformaldehyde. The fixative was removed, cells were washed twice, and subsequently permeabilized for 5 min using $0.1 \%$ Triton-X in $1 \mathrm{X}$ PBS. Cells were then blocked with $1 \%$ bovine serum albumin (Sigma-Aldrich; Merck KGaA, Darmstadt, Germany) in $1 \mathrm{X}$ PBS for $20 \mathrm{~min}$. After washing, cells were stained for 30 min with Alexa Fluor ${ }^{\mathrm{TM}} 488$ Phalloidin (Thermo Fisher Scientific, Inc.), by adding $100 \mu \mathrm{l}$ of the working stain solution $(0.165 \mu \mathrm{M})$ to each well in the dark. The cells were washed twice, and counterstained with Hoechst 33342 for 15 min by adding $100 \mu \mathrm{l}$ of the working stain solution $(1 \mu \mathrm{g} / \mathrm{ml})$ to each well in the dark. Stained cells were mounted in 1X PBS and sealed with a cover slip. Slides were observed under a fluorescence microscope (Olympus FSX100" ${ }^{\mathrm{TM}}$; Olympus Corporation, Tokyo, Japan) at 400x magnification, using the green fluorescent filter ( $\lambda \mathrm{ex} / \lambda \mathrm{em}: 490 / 525 \mathrm{~nm})$ to visualize stained filamentous actin structures, and the blue fluorescent filter ( $\lambda$ ex/ $/ \lambda \mathrm{em}: 355 / 465 \mathrm{~nm})$ to visualize the nuclei.

Scratch wound assay. Cells were seeded and transfected with a pTargeT ${ }^{\mathrm{TM}}$ KRAS construct (wild-type or mutant) in 12-well plates as previously described. Upon reaching full confluence, the cell monolayer was scratched with a sterile white pipette tip, washed with $1 \mathrm{X}$ PBS, and fed with serum-depleted media $(2.5 \%$ BCS). Bright field and fluorescence images of the same gap area were captured in 4-h intervals starting immediately after the scratch was made. The rate of wound closure was quantified over a 16-h interval, at which point the gaps were still defined by drawing parallel lines along the cell migration front and measuring the difference in gap distance using imaging software (Olympus DP2-BSW; Olympus Soft Imaging Solutions $\mathrm{GmbH}$, Münster, Germany). Additional scratch wound assay experiments were performed to visually track migrating cells. In these parallel experiments, cells were cotransfected with each pTarge ${ }^{\text {тм }}$ construct and pmR-ZsGreen 1 in a 1:7 vector ratio (pmiR-ZsGreen1: pTargeT) as previously described and wound gaps were observed over a 40-h interval, to track the expression of the fluorescent reporter.

Cell proliferation assay. CellTiter $96^{\circledR}$ AQueous One Solution Cell Proliferation Assay (Promega Corporation) was used to measure the rate of cell proliferation. Transfected cells in 12 -well plates were trypsinized at $37^{\circ} \mathrm{C} 24 \mathrm{~h}$ post-transfection. For each transfection reaction, the detached cell suspension was split into two aliquots, and cells were collected via centrifugation at $400 \mathrm{x} \mathrm{g}$ at room temperature for $2 \mathrm{~min}$. One half of the cells was resuspended in complete, serum-rich media $(10 \%$ $\mathrm{BCS})$, while the other half was resuspended in serum-depleted media $(2.5 \%$ BCS). The cells in both suspensions were stained at room temperature with trypan blue for $5 \mathrm{~min}$ and subsequently quantified using a hemocytometer. The suspensions were diluted to a final concentration of 2,500 cells $/ 100 \mu \mathrm{l}$. An equal number of cells (i.e., 2,500) were seeded in triplicate in two 96-well plates, corresponding to two time points at which the number of viable cells was measured (i.e., 48 and $72 \mathrm{~h}$ post-transfection). The plates were incubated in a humidified atmosphere containing $5 \% \mathrm{CO}_{2}$ at $37^{\circ} \mathrm{C}$ and processed 48 and $72 \mathrm{~h}$ post-transfection. To process a plate for spectrophotometric analysis, $10 \mu \mathrm{l}$ of CellTiter $96^{\circledR}$ AQueous One Solution was added to each well followed by a $2 \mathrm{~h}$ incubation to facilitate color development. Simultaneously, wells with predetermined cell counts were also prepared to serve as standards. Absorbance value of each well was measured at $490 \mathrm{~nm}$ by a colorimetric plate reader (FLUOstar Omega Microplate Reader, BMG LABTECH, Cary, NC, USA). A standard curve showing the linear relationship between absorbance value and cell count was generated and used to quantify the number of viable cells in each well. The cell counts of all transfectants were compared at both time points and statistically analyzed.

ELK1, ETS transcription factor-transactivation domain (ELK-TAD) luciferase reporter assay. The 293 cells with an ELK-TAD luciferase reporter assay system (Signosis Inc., Silicon Valley, San Francisco, USA; cat. no. SL-0040-FP) was used to measure the ability of the KRAS mutants to activate the mitogen-activated protein kinase (MAPK) signaling pathway. The cell line is stably integrated with a fusion protein of the ELK-transactivation domain and Gal4. Activation of ELK-TAD through the MAPK pathway drives the expression of firefly luciferase, which is regulated by Gal4-UAS. Cells were maintained in DMEM supplemented with $10 \%$ fetal bovine serum (FBS; Gibco; Thermo Fisher Scientific, Inc.) and $100 \mathrm{U} / \mathrm{ml}$ penicillin/streptomycin, then incubated in a humidified atmosphere containing $5 \% \mathrm{CO}_{2}$ at $37^{\circ} \mathrm{C}$. For the luciferase assay, 10,000 cells were seeded into each well of a 96-well plate. Cells were then transfected with $200 \mathrm{ng}$ of the pTargeT-KRAS constructs per well using Lipofectamine $^{\mathrm{TM}}$ 2000. Twenty-four hours post-transfection, the media was changed to DMEM supplemented with $4 \%$ FBS to serum deprive the cells, which were then allowed to grow a further $24 \mathrm{~h}$ prior to the assay. Cells were lysed by adding $20 \mu \mathrm{l}$ of Passive Lysis Buffer (cat. no. E1941; Promega Corporation) into each well, followed by a 20 -min incubation at room temperature. The cell lysates were transferred into an opaque 96-well white plate, and $100 \mu \mathrm{l}$ of the Luciferase Assay Reagent (Promega Corporation; cat. no. E1483) was added into each well. The plate was read using a luminometric plate reader (FLUOstar Omega Microplate 
Reader, BMG LABTECH) under the pre-set Firefly luciferase settings. Readings were normalized against the relative cell count measured through fluorescent staining of the cells' DNA using the CyQUANT NF Cell Proliferation Assay kit (Thermo Fisher Scientific Inc.; cat. no. C35007), according to the manufacturer's protocols, on transfected cells in parallel wells. Cells in these wells had their media removed, and $100 \mu \mathrm{l}$ of the CyQuant NF reagent was added per well. The plate was then incubated at $37^{\circ} \mathrm{C}$ for $1 \mathrm{~h}$. Fluorescence measurements were performed using a plate reader (FLUOstar Omega Microplate Reader, BMG LABTECH) with an excitation wavelength of $485 \mathrm{~nm}$, and emission detection at $530 \mathrm{~nm}$.

Bioinformatics-based prediction of potentialfunctionalimpact of mutations. The functional impact of KRAS E31D and E63K mutations was assessed using three sequence-based prediction platforms: Polymorphism Phenotyping (POLYPHEN-2; version 2) (22), Sorting Intolerant From Tolerant (SIFT; version 5.2.2) (23) and Mutation Assessor (release 2) (24).

The impact of the amino acid changes on KRAS protein structure was evaluated by building homology models of the mutants based on the solved 3D structure of human KRAS (PDB: 3GFT). As 3GFT harbors the Q61H mutation, the wild-type structure was initially prepared by reverting this activating mutation in silico. The mutants were built in Accelrys Discovery Studio Client 2.5 (Dassault Systèmes BIOVIA, San Diego, CA, USA) using the MODELER protocol, which scores and optimizes the conformation of a mutated residue and its neighbors within a defined structure (15). The global main chain root-mean-square distance (RMSD) of each mutant model was then computed based on sequence alignment with the wild-type.

The putative effect of the mutants on GTP binding was assessed by simulating ligand-receptor interactions. First, the GTP ligand was prepared by generating multiple docking poses based on optimum conformations, tautomers, and isomers at the physiological pH. Similarly, the mutated KRAS structures were prepared using a general protocol that cleans the protein structure, removes water molecules, and optimizes the side-chain conformation for residues within the binding site (25). A minimization step was also performed to the cleaned protein models in order to minimize the energy of the structure, relax the protein conformation, and remove steric overlaps that may produce inaccurate interactions. Using the binding site coordinates defined by the reference solved crystal structure, the generated ligand poses were docked within the binding sphere of each KRAS protein variant. The CDOCKER algorithm was used to generate the docked conformations of the ligand (26). The calculated docking scores were compared among the different forms of KRAS.

Statistical analysis. All quantitative data were presented as mean \pm standard error. One-way analysis of variance with post hoc Tukey's Honest Significant Differences was performed to compare and evaluate the quantitative results of the cellular assays. A P-value of 0.05 was used for all assays.

\section{Results}

Rare mutants E31D and E63K induce cell rounding, refringency, and cytoplasmic shrinkage. The flattened phenotype of normal non-transformed NIH3T3 fibroblasts was predominant in cells transfected with the empty expression vector. Non-transformed cells are reasoned to be flat and well-spread because of the appropriate and well established adhesion of cells onto the culture substrate (27). On the other hand, transformed NIH3T3 cells are characteristically smaller, rounder, and highly refractile compared with their non-transformed counterparts $(28,29)$. Transformed cells also possess pronounced pseudopods and cellular protrusions (8). These characteristics likely reflect cytoskeletal reorganization resulting in poor surface adhesion and promotion of cellular motility.

Compared with the vector control, no significant morphological changes were seen in cells overexpressing the wild-type protein (Fig. 1). This observation is corroborated by previous reports that amplified expression of normal RAS is usually not sufficient for cellular transformation and therefore produces no discernible morphological changes in NIH3T3 cells $(28,29)$.

As expected, a significant fraction of cells transfected with constitutively active forms of KRAS, including KRAS G12D and KRAS G13D, showed morphological features that mirror those of transformed fibroblasts, as the cells became smaller, rounder, and more refringent (Fig. 1). Cytoplasmic shrinkage was also apparent in the transfected fibroblasts. A significant percentage $(\mathrm{P}<0.05)$ of cells transfected with either the KRAS E31D (33\%) or KRAS E63K (38\%) mutant also share the morphology typical of transformed cells, hinting at the transformation potential of the rare mutations. While equal cell densities among the treated cells could not be guaranteed at the observation time-point, considering the combined cytotoxic and proliferative effects of transfection and KRAS overexpression, respectively, the trend of increased morphological aberration in KRAS-mutant overexpressing cells was highly reproducible. In all our experiments it was also ensured that morphology assessment was performed before cells reached full confluence and that any perceived differences in cell densities can be attributed to the aforementioned morphological changes.

Cytochemical staining of filamentous actin in cells overexpressing KRAS mutants reveals altered cytoskeletal organization. Activating $R A S$ mutations are known to affect actin organization, consequently facilitating the onset of cellular transformation (30). To determine if the rare KRAS mutations under study have the same deleterious impact on cytoskeletal dynamics similar to canonical mutants, filamentous actin of transfected cells was visualized via fluorescence staining. Transfected cells cultured in 8-well chamber slides were washed, fixed and permeabilized prior to staining. High-affinity binding of phalloidin to actin was used to visualize the actin cytoskeleton. Hoechst was used to counter-stain the nuclei.

Whereas the vector control- and wild-type-expressing cells exhibited prominent and well-oriented stress fibers, all canonical- and rare mutant-expressing cells developed poorly defined and thin stress fibers (Fig. 2). In KRAS E31D- and KRAS E63K-transfected cells, as well as in the positive controls, cell depolarization was apparent with extensive membrane ruffling and lamellipodia formation around the cell periphery. Formation of filopodia and pseudopodia were also increased. Altogether, these cytoskeletal features are typical of contractile and motile cells with highly dynamic actin networks, suggesting the effect of the rare KRAS mutants on cellular migration. 


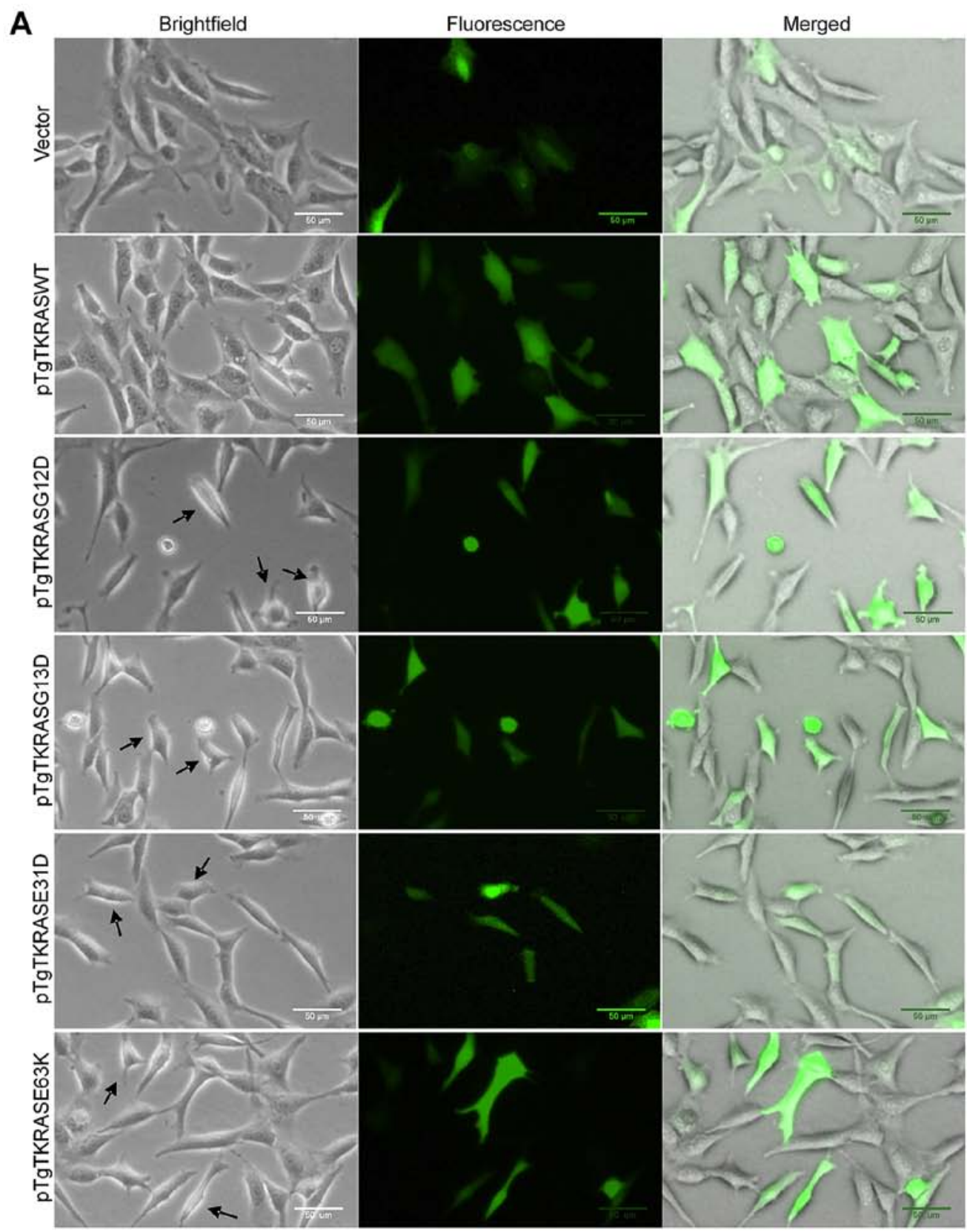

B

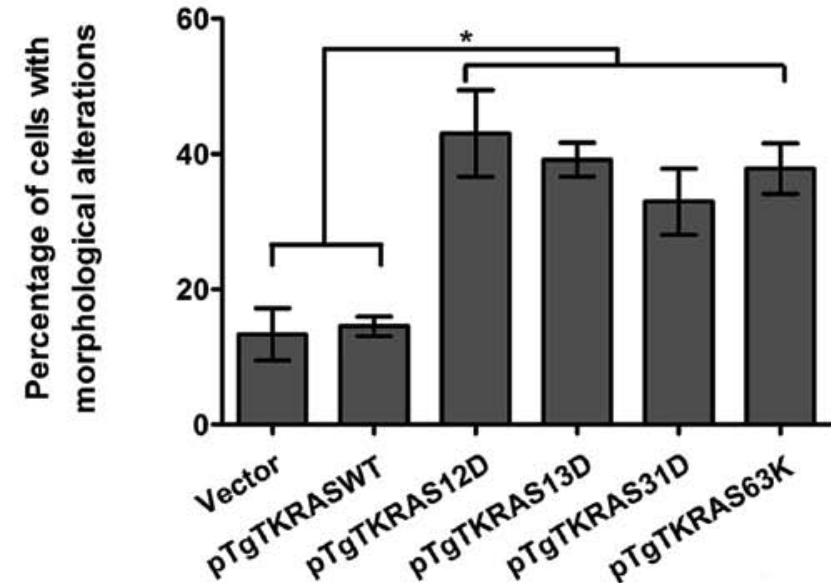

Figure 1. KRAS E31D- and KRAS E63K-transfected cells show altered gross cellular morphology similar to canonical mutant controls (representative of three trials). (A) NIH3T3 cells were co-transfected with a pTargeT ${ }^{\mathrm{TM}}$ construct and empty pmiR-ZsGreen1, the latter to visualize transfected cells. The black arrows point to KRAS mutant transfectants with altered cell morphology. Scale bars: $50 \mu \mathrm{m}$. (B) NIH3T3 cells overexpressing canonical and novel KRAS mutants that exhibit apparent morphological alterations were counted and divided by the total number of fibroblasts in each view. The fraction of cells with morphological irregularities was significantly greater in populations overexpressing a mutant variant of the protein. "P $<0.01$. WT, wild-type. KRAS, KRAS Proto-Oncogene, GTPase. 


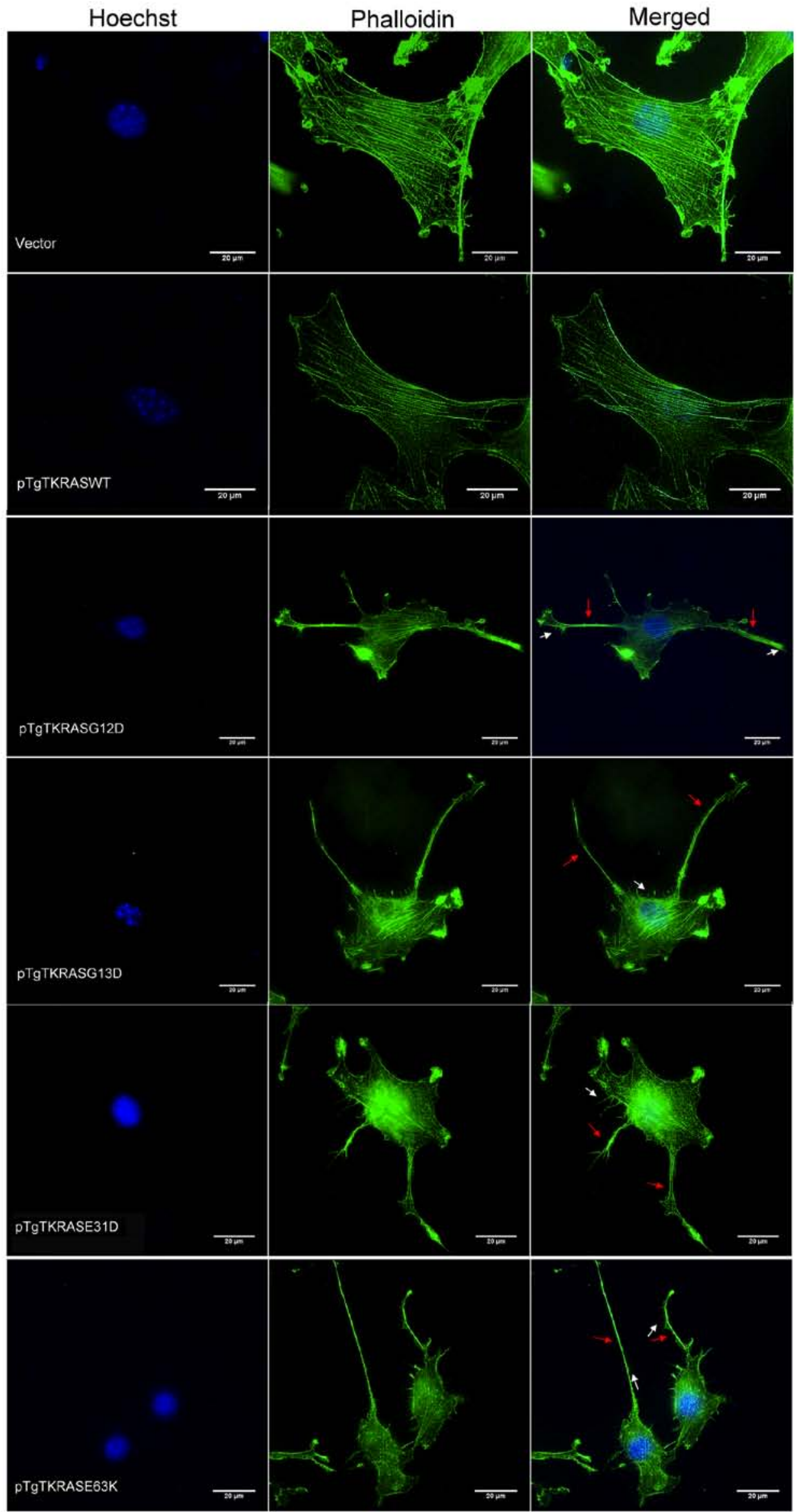

Figure 2. Cytoskeletal features associated with motility are prominent in cells transfected with KRAS E31D and KRAS E63K. Red arrows indicate polymerized actin of pseudopod extensions in cells transfected with the canonical (pTgTKRASG12D and pTgTKRASG13D) and novel KRAS (pTgTKRASE31D and pTgTKRASE63K) mutants. White arrows indicate filopodial actin. In addition to an overall diffused cytoskeletal actin, emergence of pseudopods and filopodial structures were observed in cells transfected with mutant KRAS constructs. KRAS, KRAS Proto-Oncogene, GTPase. 

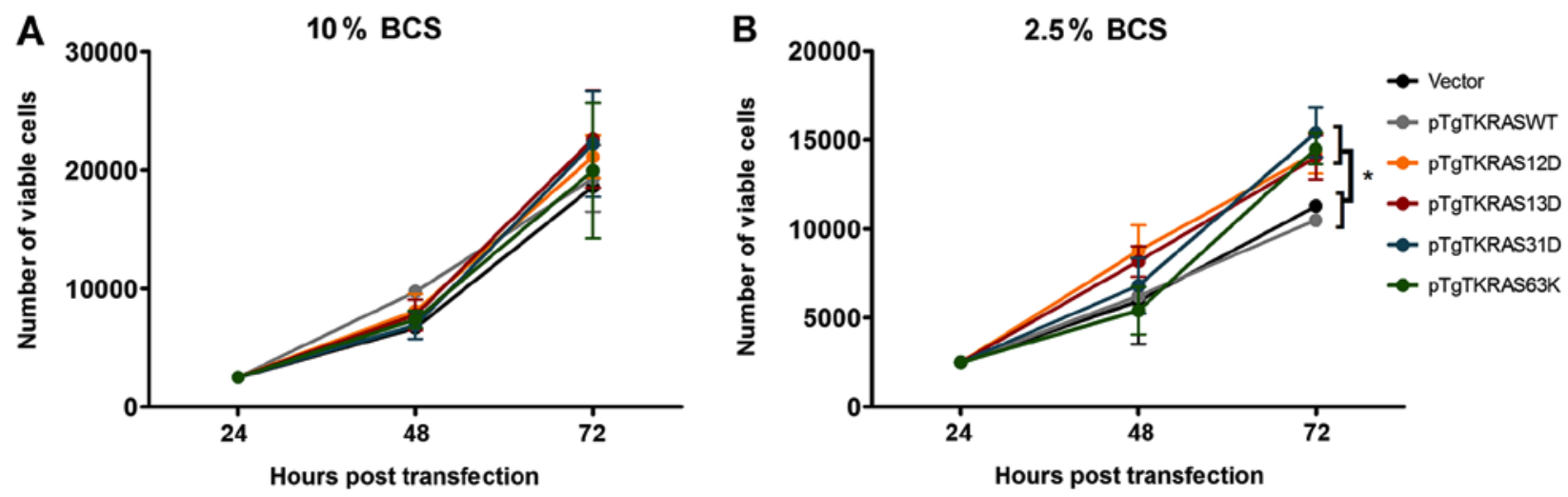

Figure 3. NIH3T3 cells transfected with canonical and novel KRAS mutants increased cellular proliferation (representative of three trials). Experiment showing the effect of KRAS mutant overexpression on cell proliferation. (A) Cells fed with $10 \%$ serum showed comparable proliferation capacity. (B) Cells transfected with mutant KRAS, canonical and novel, showed significant increase in proliferative capacity at 72-h post-transfection when grown in serum-depleted conditions. ${ }^{*}$ < 0.01 . BCS, bovine calf serum; WT, wild-type; KRAS, KRAS Proto-Oncogene, GTPase.

KRAS E31D and E63K mutations confer increased proliferative capacity. The proliferation rates of cells transfected with the KRAS E31D and KRAS E63K mutants were assessed and compared with the vector, wild-type, and canonical mutant controls (Fig. 3). This was done by seeding an equal number of cells that have been transfected for $24 \mathrm{~h}$ onto 96 -well plates in triplicates and quantifying the number of viable cells via a colorimetric reagent 48 and $72 \mathrm{~h}$ post-transfection. At high serum concentration (10\%), all transfectants showed comparable number of viable cells at the observed time points (Fig. 3A). In contrast, in serum-depleted (2.5\%) conditions, cells expressing KRAS G12D, KRAS G13D, KRAS E31D, and KRAS E63K all showed a significant increase in cell count $72 \mathrm{~h}$ post-transfection (Fig. 3B). The assays also indicated that the absolute number of viable cells was, as expected, dependent on serum concentration.

Cells expressing canonical and rare KRAS mutants show increased cell migration rates in scratch wound assays. Given the impact of KRAS E31D and KRAS E63K overexpression on cellular morphology and cytoskeletal actin, scratch wound assays were performed to investigate cell motility and its association with the expression of the rare mutants. The transfected cell monolayers were grown to full confluence, upon which a scratch was introduced using a sterile pipette tip. The cultures were then shifted to serum-depleted media (2.5\%) and the wound fields were observed in a time-course manner. In some trials, pmR-ZsGreen1 was co-transfected with the expression vectors to ascertain, through expression of the fluorescent protein, whether migrating cells were positively transfected or otherwise.

Wound gaps were routinely observed at 4-h intervals until the confluent monolayer for each transfected culture was restored. As it became difficult to delineate the opposing migrating fronts beyond a certain time point, as cells started to extensively populate the gap wound from either side, the rate of wound closure was calculated $16 \mathrm{~h}$ post-scratch (Fig. 4A). The gap closure rates in cells expressing KRAS E31D and KRAS E63K mutants were significantly higher $(\mathrm{P}<0.05)$ compared with the vector and wild-type controls, and comparable with cells expressing the constitutively active forms of KRAS (Fig. 4B). Further, in co-transfected setups, fluorescent cells were particularly prominent midway the closing gap for cultures transfected with either KRAS E31D or KRAS E63K (Fig. 4C). The same was observed for cultures transfected with either canonical mutant. Although non-fluorescent cells were also present in the gap it is highly likely that these cells harbor the KRAS construct regardless, since cells were transfected with a 1:7 vector ratio (pmiR-ZsGreen1: pTargeT). Altogether, these results imply that positively transfected cells were more likely to migrate into the gap, suggesting the functional consequence of the mutations on cell motility.

In contrast, at $40 \mathrm{~h}$ following wounding, even fewer fluorescent cells were spotted halfway into the scratch wound of cells transfected with either the empty vector or wild-type KRAS, and a conspicuous gap could still be seen under the green fluorescent filter. Because transfection efficiency was consistent among treatments based on fluorescent quantification using fluorescence microscopy (data not shown), this underscores the comparable tendency for migration of empty vector- and KRAS-WT-transfected cells, adding evidence to the limited transforming effect of wild-type KRAS overexpression. For the empty vector and wild-type KRAS-transfected cultures, non-fluorescent cells were able to migrate into the gaps, due to the relatively long observation period. These cells most likely reached their terminal positions at reduced velocities, as indicated by the data on wound closure rates.

Effect of KRAS mutations on ELK transactivation. To determine whether KRAS E31D and KRAS E63K have an effect on downstream targets, an ELK-TAD luciferase reporter assay was employed. ELK-1 is an established nuclear target and one of the best characterized substrates of ERK, a downstream effector of KRAS $(31,32)$. The different KRAS constructs were transfected into a 293 cell line harboring a stably integrated ELK/luc reporter to monitor their effects on ELK-1 transactivation. As shown in Fig. 5, the canonical mutants KRAS G12D and KRAS G13D, as well as the novel mutant KRAS E63K, consistently stimulated ELK-1-mediated transcriptional response. KRAS E31D, on the other hand, only showed stimulation of ELK-1 in two out of three trials and only achieved significance in one trial $(\mathrm{P}<0.05)$. As discussed below, in silico analyses suggested that the effects 
A

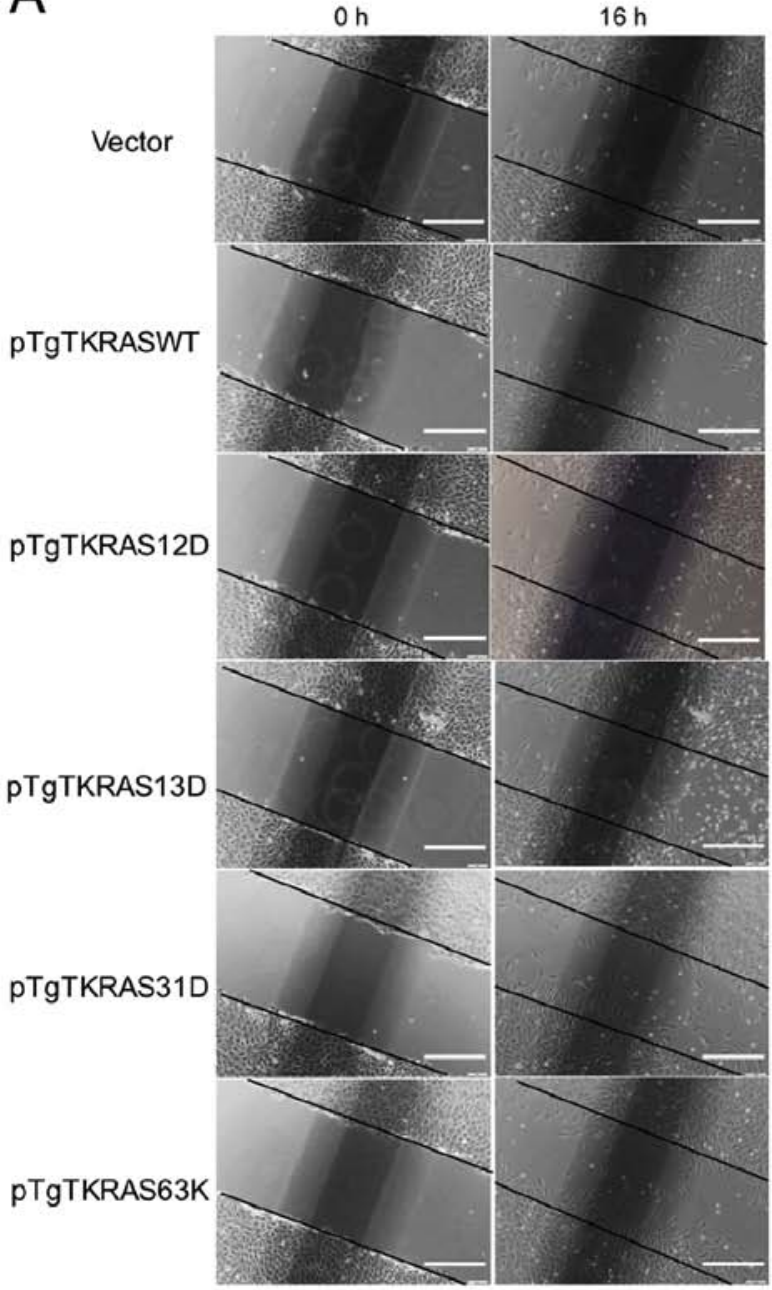

B

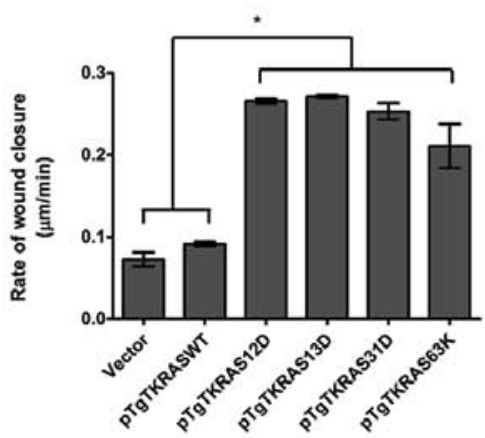

C

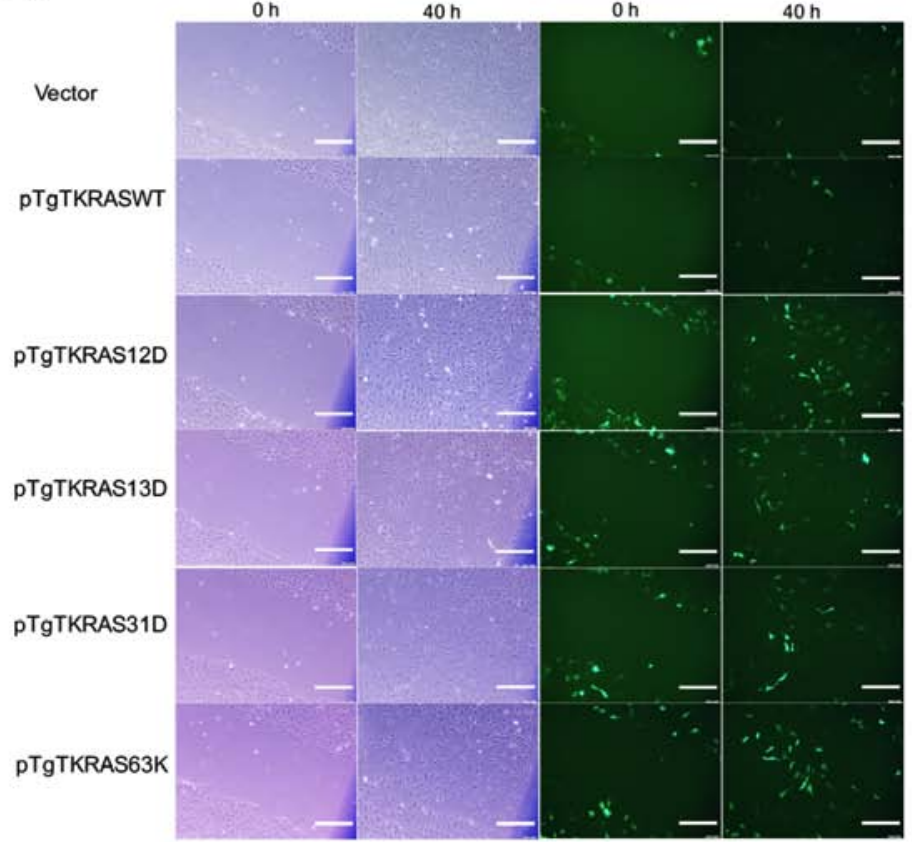

Figure 4. Overexpression of canonical and novel KRAS mutants resulted in increased cell migration in vitro. Scratch wound assays were conducted using transfected NIH3T3 cells grown in $2.5 \%$ serum. (A) Micrographs of wound fields directly after scratching the monolayer (0 h), and $16 \mathrm{~h}$ post-scratch. Compared with the controls (i.e., vector, pTgTKRASWT), narrower scratch gaps were noticeable for cells overexpressing KRAS mutant variants. Scale bars: $100 \mu \mathrm{m}$. Representative of three trials. (B) The distance between lines approximating the cell migration front was measured for each setup at time points 0 and $16 \mathrm{~h}$, and the rate of front migration was calculated. Wound closure was significantly faster for cells overexpressing the mutant protein. ${ }^{*} \mathrm{P}<0.01$. (C) $\mathrm{NIH} 3 \mathrm{~T} 3$ cells positively transfected with mutant KRAS constructs have an increased tendency to migrate into wound gaps. Cells used for scratch wound assays were co-transfected with a transfection marker, pmiR-ZsGreen1. Migrating fibroblasts were tracked directly after scratching the monolayer ( $0 \mathrm{~h})$, and $40 \mathrm{~h}$ post-scratch to verify that cells facilitating the gap closure are positively transfected. Scale bars: $100 \mu \mathrm{m}$. WT, wild-type; KRAS, KRAS Proto-Oncogene, GTPase.

of this mutation may be benign. However, given the consistent and comparably aggressive phenotypic readout of this mutant compared with KRAS G12D and KRAS G13D in the different cancer hallmark assays, this may mean that KRAS E31D mediates its effects not through ELK-1, but via another downstream target.

Functional consequences of KRAS E31D and KRAS E63K mutations as predicted through in silico protein modelling and docking simulations. The effect of E31D and E63K mutations on KRAS GTPase function was predicted using three bioinformatics platforms: PolyPhen-2 (22), which utilizes a physical and comparative approach in relating structure to function; SIFT (23), which yields predictions based on sequence homology and physical properties of pertinent amino acids; and Mutation Assessor (24), which relies on the evolutionary conservation of the mutated amino acid in protein homologs. In all platforms, E31D was predicted to have null to minimal effect on protein function, whereas E63K scored high ratings of potential functional impact in PolyPhen-2 and SIFT (Table I). However, these predictions only analyze the protein in isolation, precluding the possible effects of a mutation on the binding of regulators, including adaptors, scaffolds, and other proteins in a signalosome, in addition to effectors.

Similar to the canonical mutants KRAS G12D and KRAS G13D, both KRAS E31D and KRAS E63K have minimal effect on the overall structure of KRAS, based on the calculated RMSD values (Fig. 6). However, all mutants resulted in apparent conformational changes in a region critical to the GTPase activity of the protein, the mobile loop switch I, whose relative orientation dictates the activity status of KRAS. In both KRAS E31D and KRAS E63K, noticeable repositioning 
Table I. Functional effects of KRAS E31D and E63K as predicted by multiple bioinformatics platforms.

\begin{tabular}{llll}
\hline Mutation & \multicolumn{1}{c}{ Polyphen-2 (score) } & \multicolumn{1}{c}{ SIFT (score) } & Mutation assessor (score) \\
\hline E31D & Benign $(0.000)$ & Tolerated $(0.06)$ & Neutral (0.49) \\
E63K & Probably damaginga $(0.985)$ & Affect protein function $(0.00)$ & Low (1.485) \\
\hline
\end{tabular}

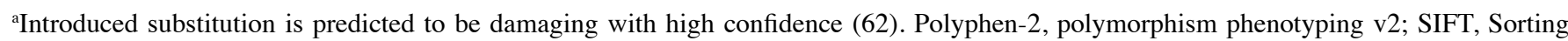
Intolerant From Tolerant; KRAS, KRAS Proto-Oncogene, GTPase.

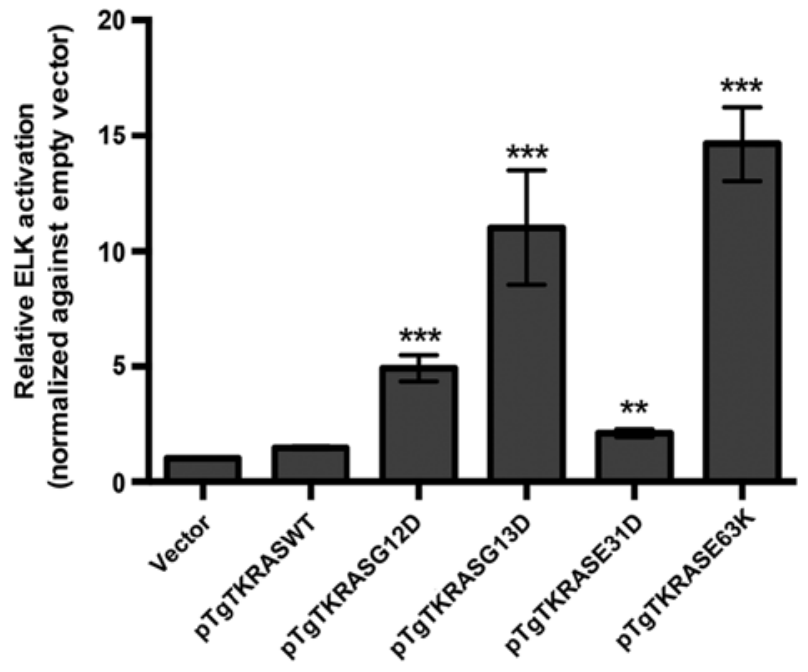

Figure 5. ELK-TAD luciferase reporter assay showed increased activation of ELK-1 by KRAS mutants. ${ }^{* *} \mathrm{P}<0.001$ and ${ }^{* * *} \mathrm{P}<0.0001$. ELK-1, ELK1, ETS transcription factor; WT, wild-type; KRAS, KRAS Proto-Oncogene, GTPase.

of the switch I loop was observed. The canonical mutants KRAS G12D and KRAS G13D resulted in a reorientation of the same loop. Perturbations caused by the canonical mutants are corroborated by crystal structures solved previously (33).

Docking simulations using CDOCKER were performed to determine the effect of mutations on ligand binding. CDOCKER functions on an algorithm wherein the receptor is held rigid while the ligand is allowed to flex during pose refinement. The homology mutant models of KRAS E31D, E63K, G12D, and G13D were prepared and minimized prior to docking. A total of 64 poses of the pH-optimized GTP ligand were generated for the simulations, with considerations to possible conformations, tautomers, and isomers. For each variant of KRAS, 192 GTP-receptor complex poses were scored. As shown by the minimum docking scores, KRAS G13D had the most favorable interaction with GTP, followed by the wild-type, E31D, E63K, and finally, the G12D mutant (Fig. 7). The exergonic CDOCKER energy values for all KRAS variants predicted unimpeded and highly favorable ligand binding. In all cases, the highly negative triphosphate moiety of GTP was correctly oriented toward the positively charged metal cofactor within the binding pocket.

\section{Discussion}

Based on the varying epidemiological distribution of mutations reported in different countries, KRAS mutation frequency and spectrum are most likely dependent on the population under study $(14,18)$. Even with the 2-4-fold increase in the incidence of the disease in Asian countries, molecular statistics considering the regional population is lacking (18). It is therefore paramount to investigate rare and novel KRAS mutations detected in the local population in association with their impact on CRC diagnostics, prognostics, and therapeutics.

Another reason for examining the functional consequences of novel KRAS mutations is intratumor heterogeneity. This suggests that cancer cell clones coexisting within a tumor present a divergent set of genetic aberrations (34). Cells with similar phenotypes may also segregate and enrich a particular region of the tumor, leading to sampling bias and subsequent underestimation of important mutations and biomarkers (35). The genetic variation within a tumor has also been cited as a cause of targeted drug resistance: Low-frequency cells carrying resistance-conferring mutations eventually outgrow the dominant clones susceptible to the therapy $(34,36)$. Taking the aforementioned into consideration, the presence of rare mutations could ultimately be predictive of treatment outcome.

Further underscoring the significance of low-frequency $K R A S$ mutations in CRC is a recent report on the non-responsiveness of patients harboring mutations beyond the canonical hotspots to cetuximab treatment $(37,38)$. Although a definitive correlation could not be established between the occurrence of the reported rare mutations and refractory cancer due to low patient numbers, there is sufficient evidence to suggest that these mutations are significant in predicting tumor susceptibility to anti-EGFR treatment $(39,40)$.

The functional impact of two previously reported KRAS mutations was assessed in this study. The first mutation, E31D in exon 2 (NM_004985.3: c.93A>T; p.31E>D), was surveyed from a genetic screen of histopathologically positive CRC tissues from Filipino patients at the Philippine General Hospital. Alternative codon 31 mutations in KRAS have also previously been reported in CRC (41), papillary thyroid carcinoma (42), and adrenal tumors (43). To the best of our knowledge, the functional effects of these mutations have yet to be investigated. The other mutation, E63K in exon 3 (NM_004985.3: c.187G>A; p.63E $>\mathrm{K}$ ), is curated in the COSMIC database and has been reported to occur in lesions of the large intestine, genital tract, thyroid, lymphoid tissue, and medulla (19). Eight separate E63K mutations were cited in COSMIC, and three were identified in CRC (19). The results obtained in the current study demonstrated that, the E63K mutation scored high ratings of potential functional impact from two predictive amino acid substitution tools (i.e., PolyPhen-2 and SIFT). Despite the rarity of these KRAS mutations, the aforementioned reports suggest the significance 

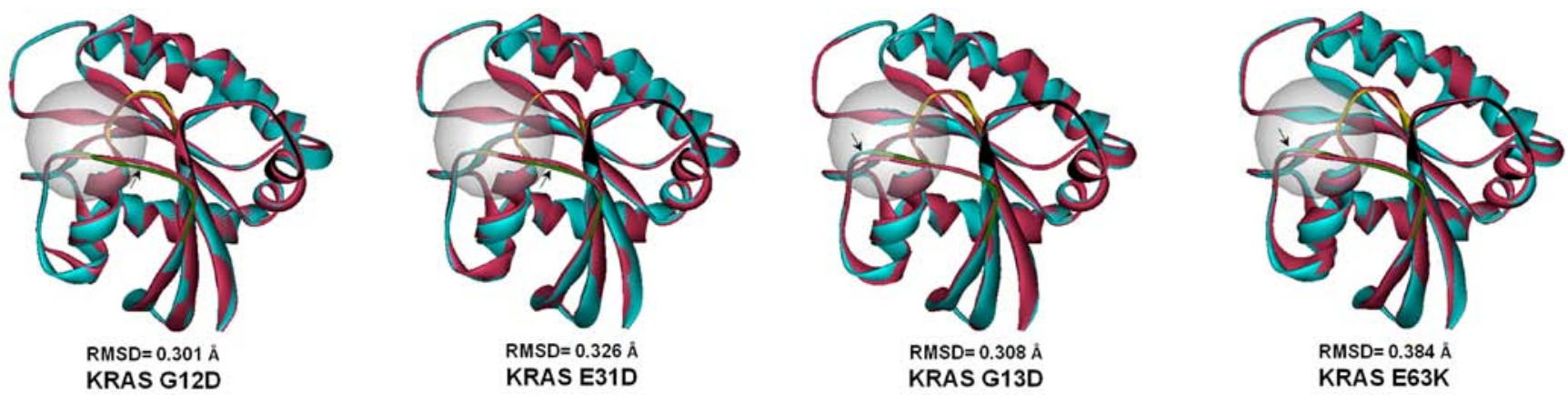

Figure 6. Superposition of modeled KRAS mutants with the solved crystal structure of human KRAS showed structural perturbations introduced by E31D and E63K mutations in a region critical to GTPase activity (PDB ID: 3GFT; cyan ribbon). Canonical and novel mutants are represented in red In each overlay image, the sphere represents the GTP-binding site of KRAS. Critical residues of the protein are highlighted in the wild-type schematics as follows: Yellow, phosphate binding-loop; green, switch I; black, switch II. Apparent deviations in the protein conformation brought about by the single amino acid mutations are denoted by arrows. Global RMSD values were also predicted via sequence alignment function for each mutant in comparison with the wild-type. RMSD, root-mean-square distance; KRAS, KRAS Proto-Oncogene, GTPase.

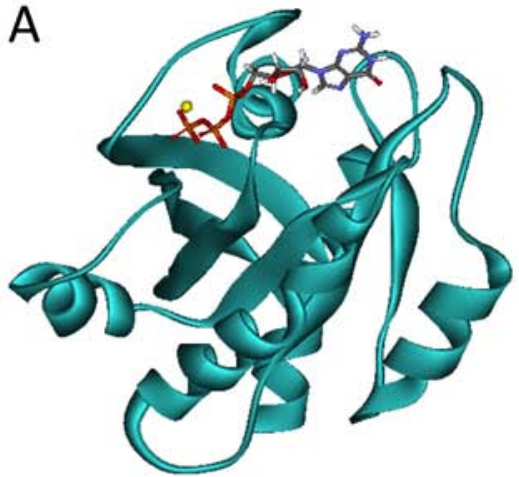

CDOCKER energy $=-66.7209 \mathrm{kcal} / \mathrm{mol}$ KRAS WT

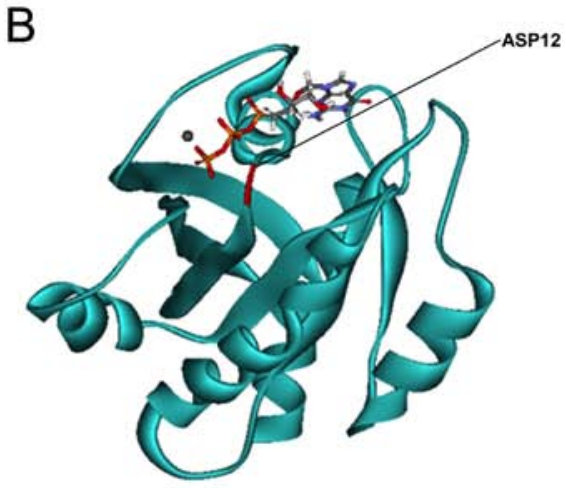

CDOCKER energy $=-24.4063 \mathrm{kcal} / \mathrm{mol}$ KRAS G12D

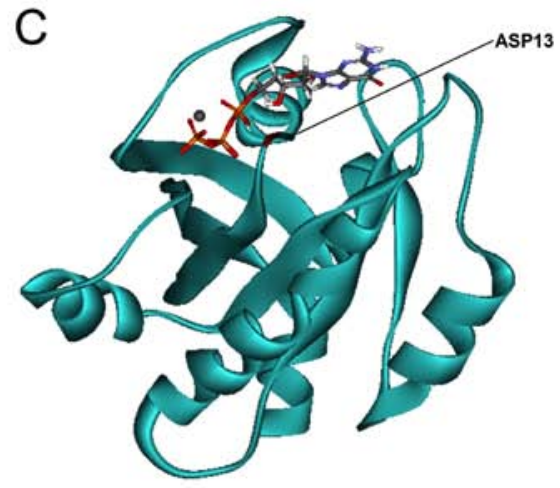

CDOCKER energy $=-77.0621 \mathrm{kcal} / \mathrm{mol}$ KRAS G13D
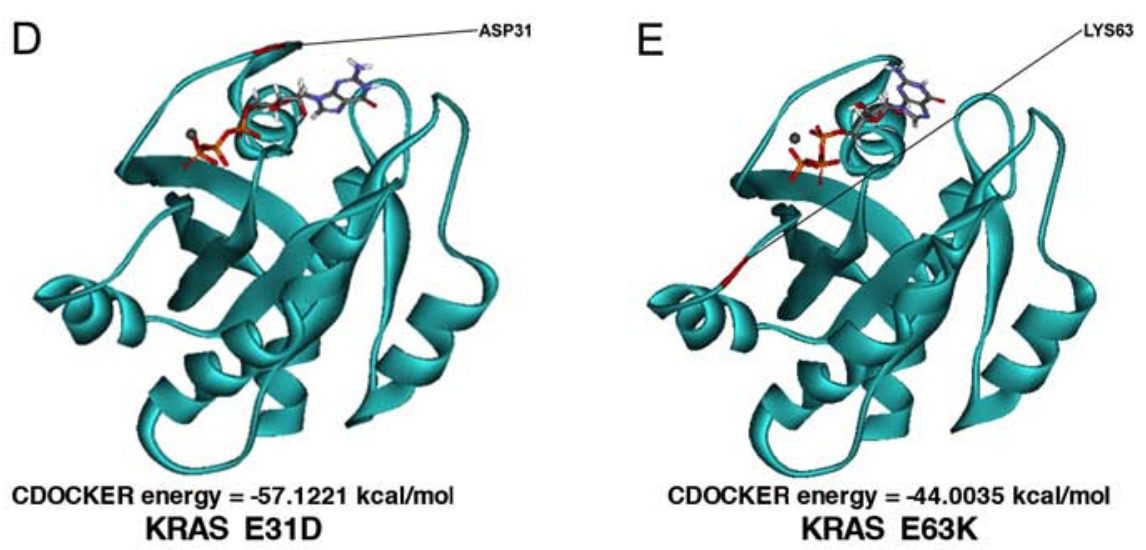

Figure 7. Simulated interactions of GTP to wild-type and mutant KRAS yielded variable binding energies. The solved x-ray crystal structure of human KRAS. (A) PDB: 3GFT was used to build mutant homologues (B) G12D, (C) G13D, (D) E31D and (E) E63K. GTP binding was implemented using the CDOCKER algorithm. The ligand conformation with the lowest (i.e., most negative) CDOCKER energy is shown for each interaction diagram. For the mutant proteins, the amino acid change is highlighted in red and labelled accordingly. The magnesium ion cofactor is shown as a grey sphere above the bound GTP. WT, wild-type; KRAS, KRAS Proto-Oncogene, GTPase.

of amino acid positions 31 and 63 in the normal cycling of the protein, and the potential transformative effects brought about by mutations in the two loci.

In the present study, the transforming potential of the rare KRAS mutations E31D and E63K were revealed based on morphological and functional characterization of NIH3T3 cells overexpressing the mutant oncoproteins. The murine embryonic fibroblast NIH3T3 were selected as the heterologous host, due to its well documented phenotypic response upon overexpression of activating RAS genes (44-48). Unlike other common CRC lines including HCT-116 (G13D), DLD-1 (G13D), SW480 (G12V), and SW948 (Q61L), NIH3T3 has a wild-type KRAS background, which is necessary for the evaluation of putative activating mutants. Finally, unlike a number 
of cell lines and primary cultures that require complementation of cooperative oncogenes, NIH3T3 cells readily exhibit oncogenic phenotypes upon lone expression of a transforming $R A S$ variant (49). A caveat in our approach to functionalize the novel mutants is the use of a transient overexpression system in the cellular assays. To mitigate the potential confounding effects of inconsistent transfection efficiencies, the robustness of the procedure was ensured by co-transfection with pmiR-ZsGreen1, which encodes a green fluorescent protein marker, followed by assessment by fluorescence microscopy.

Cells overexpressing the rare mutants were indicated to share phenotypic features with G12D or G13D-transfected cells in terms of morphology, actin cytoskeleton organization, proliferative capacity, and migration rate, validating the hypothesis that the rare mutants could induce cellular transformation. The non-transforming effect of wild-type KRAS transient overexpression was also observed in the cellular assays, which was in accordance with previous reports $(28,29)$.

Enhanced cell motility by E31D and E63K overexpressing cells was only observed in serum-depleted conditions. In serum-rich media, all transfected cells showed comparable rates of wound gap closure. Mitogens, cytokines, and growth factors present in the serum may have saturated cell surface receptors, which were significant to effector pathways associated with cell motility and proliferation, including members of the ErbB family of receptors (50). On the other hand, utilizing serum-depleted media allows cells to synchronize and enter the quiescent $G_{0} / G_{1}$ phase, preventing the mistaken collection of wound gap closure data on actively proliferating cells (51). Working with a serum-depleted background ensures differential pressures of the wild-type and mutant KRAS oncoproteins on associated signaling pathways, without the unwanted contribution of excess growth factors.

The majority of viable cells observed for both E31D and E63K transfectants was further evidence of the transforming potential of the rare mutants. This increase in proliferative capacity may be attributed to the activation of the Raf-MEK-ERK signaling pathway resulting from ectopic expression of either KRAS E31D or E63K. Parallel experiments performed in high-serum conditions yielded comparable cell counts across all transfectants at the same time points, underlining the importance of the background medium in obtaining biologically significant readout from such sensitive assays. Notable differences in cell counts were observed only upon reaching the 72-h post-transfection time point, suggesting the time required to accelerate the mobilization of molecular and cellular components necessary for cell proliferation that are contingent on KRAS activation.

The ability of both canonical and novel KRAS mutants to activate ELK-1, a downstream nuclear target and well-characterized substrate of ERK, was also assessed through the ELK-TAD luciferase reporter assay. Similar to the canonical mutants KRAS G12D and KRAS G13D, the novel mutant KRAS E63K showed activation of ELK, compared with KRAS WT and vector-only control. In contrast, KRAS E31D only showed activation of ELK in two of three trials and achieved significance only in one. While this may be consistent with our in silico predictions of a benign phenotype for E31D, the results of cellular assays as described above prove otherwise. Therefore, it is reasonable to speculate that KRAS E31D may be mediating its effects via a different downstream effector. ERK has a plethora of downstream targets, including transcription factors c-Fos (52), c-Jun (53), cytoskeletal elements (54) and intercellular domains of membrane receptors (55). Therefore, it may be more instructive to assess the effect of the novel KRAS mutants on the more upstream effectors MEK and ERK, through phosphorylation assays. Given that KRAS also signals via at least two other pathways implicated in colorectal cancer, the effects of the mutants on the PI3K-AKT-mTOR and RAL-GEF pathways should also be assessed $(56,57)$.

The present study examined the possibility of structural changes elicited by the non-hotspot mutants on the KRAS protein by performing mutation modeling and simulating protein-ligand docking. Based on the generated global RMSD values, incorporation of either E31D or E63K mutation elicits minimal changes on the overall structure of KRAS. The constancy of the phosphate binding loop structure also indicates intact GTP-binding capability of the mutants. However, in silico analysis revealed perturbations introduced by the rare mutations in the switch I loop. The spatial influence of E31D on switch I may stem from its position near the N-terminal flank of the loop, while the positively charged amine side chain of E63K, as part of the switch II loop, could introduce a change in the local electrostatic environment that may affect the conformation of switch I indirectly. It is speculated that the altered conformation in such close proximity to the GTP-binding cleft interferes with GTP hydrolysis via steric effects.

The unique range of docking energy values generated upon simulation of GTP binding to the active site of the wild-type and mutant KRAS also hint at perturbation of protein structure. The negative CDOCKER energy values point to the spontaneous binding of GTP to the active site of wild-type and mutant KRAS. In general, the following trend in terms of favorability of binding (i.e., most negative docking score) was observed: G12D $<$ E63K $<$ E31D $<$ wild-type $<$ G13D. The trend G12D $<$ wild-type $<$ G13D was previously reported using the iGEMDOCK algorithm (58). These observations indicate that the oncogenic potential of a certain KRAS mutant does not solely rely on the strength of nucleotide binding. Although loss-of-function mutations can be identified through binding studies, the more important and more informative parameter in considering an activating mutation in KRAS, is its effect on the rate of GTP hydrolysis (33). Future docking simulations of representative GTPase-activating proteins which facilitate GTP hydrolysis, guanine nucleotide exchange factors which modulate nucleotide exchange from inactive RAS-GDP to active RAS-GTP, and other signal mediators to the KRAS mutants would provide additional insight with respect to the biochemical interactions perturbed by the amino acid alterations that ultimately lead to the observed transformed-like phenotypes in NIH3T3 cells. These aforementioned binding studies would be particularly interesting for the rare mutants, considering that amino acid positions 31 and 63 are in close proximity with the switch loops critical to GTP hydrolysis.

Scaffold proteins also spatially regulate the activation of RAS effectors by providing platforms for assembly, 
and localizing signaling molecules to specific sites in the cell $(59,60)$. A number of these proteins also protect activated signaling molecules from inactivation. For example, RAS-dependent activation of Raf in the ERK module has been shown to be accelerated, due to the binding of a scaffold protein, Shoc2/SUR-8, to RAS-GTP, reserving it for subsequent Raf interaction (61). These interactions emphasize the complex molecular mechanisms underlying signal transduction through RAS. In silico modelling of the ternary structures involving the ligand, receptor, regulators, and/or scaffolds is therefore recommended. The complete and more definitive assessment of the structural impact of the mutants can only be achieved through x-ray crystallography and nuclear magnetic resonance techniques. It is crucial that computational and predictive analyses be complemented by cellular assays, as performed in the present study, to elucidate the functional consequences of a mutation within a proper physiological context.

Lastly, the oncogenic capacity of the novel KRAS mutants E31D and E63K requires further validation through in vivo animal studies and its potential association with clinical outcomes determined, in order for them to be useful as predictive biomarkers for non-responsiveness to anti-EGFR therapy.

\section{Acknowledgements}

The authors thank Dr Junie Billones (Department of Physical Sciences and Mathematics, University of the Philippines, Manila, Philippines) for advice on protein modeling, Mr. Joshua Malapit (Disease Molecular Biology and Epigenetics Laboratory, National Institute of Molecular Biology and Biotechnology, University of the Philippines, Diliman, Philippines) for helpful discussions on protein-ligand docking, and Ms. Isabelle Viola (Disease Molecular Biology and Epigenetics Laboratory, National Institute of Molecular Biology and Biotechnology, University of the Philippines, Diliman, Philippines) for assistance with preparation of figures.

\section{Funding}

This work was supported by grants from the University of the Philippines System (grant no.06-008) and the Philippine Council for Health Research and Development (grant no. FP150025).

\section{Availability of data and materials}

The data presented in this study, including those for additional trials, are available from the corresponding author on reasonable request.

\section{Authors' contributions}

RLG and EMCDP conceived project and wrote the project proposal. AKJA and RTDY performed experiments. AKJA, RTDY and RLG analyzed and organized the data. AKJA and RLG wrote manuscript. All authors have read and approved of the final version of the manuscript.

\section{Ethics approval and consent to participate}

Not applicable.

\section{Patient consent for publication}

Not applicable.

\section{Competing interests}

The authors declare no competing financial interest.

\section{References}

1. Porru M, Pompili L, Caruso C, Biroccio A and Leonetti C: Targeting KRAS in metastatic colorectal cancer: Current strategies and emerging opportunities. J Exp Clin Cancer Res 37: 57, 2018.

2. Horsch M, Recktenwald CV, Schädler S, Hrabé de Angelis M, Seliger B and Beckers J: Overexpressed vs mutated Kras in murine fibroblasts: A molecular phenotyping study. Br J Cancer 100: 656-662, 2009.

3. Heinemann V, Stintzing S, Kirchner T, Boeck S and Jung A: Clinical relevance of EGFR- and KRAS-status in colorectal cancer patients treated with monoclonal antibodies directed against the EGFR. Cancer Treat Rev 35: 262-271, 2009.

4. Di Nicolantonio F, Martini M, Molinari F, Sartore-Bianchi A, Arena S, Saletti P, De Dosso S, Mazzucchelli L, Frattini M, Siena S and Bardelli A: Wild-type BRAF is required for response to panitumumab or cetuximab in metastatic colorectal cancer. J Clin Oncol 26: 5705-5712, 2008.

5. Baas JM, Krens LL, Guchelaar HJ, Morreau $\mathrm{H}$ and Gelderblom $\mathrm{H}$ : Concordance of predictive markers for EGFR inhibitors in primary tumors and metastases in colorectal cancer: A review. Oncologist 16: 1239-1249, 2011.

6. Prior IA, Lewis PD and Mattos C: A comprehensive survey of Ras mutations in cancer. Cancer Res 72: 2457-2467, 2012.

7. Stengel KR and Zheng Y: Essential role of Cdc42 in Ras-induced transformation revealed by gene targeting. PLoS One 7: e37317, 2012.

8. Guerrero S, Casanova I, Farré L, Mazo A, Capellà G and Mangues R: K-ras codon 12 mutation induces higher level of resistance to apoptosis and predisposition to anchorage-independent growth than codon 13 mutation or proto-oncogene overexpression. Cancer Res 60: 6750-6756, 2000.

9. Seeburg PH, Colby WW, Capon DJ, Goeddel DV and Levinson AD: Biological properties of human c-Ha-ras1 genes mutated at codon 12. Nature 312: 71-75, 1984.

10. Shankaran V, Obel J and Benson AB III: Predicting response to EGFR inhibitors in metastatic colorectal cancer: Current practice and future directions. Oncologist 15: 157-167, 2010.

11. Zhao B, Wang L, Qiu H, Zhang M, Sun L, Peng P, Yu Q and Yuan X: Mechanisms of resistance to anti-EGFR therapy in colorectal cancer. Oncotarget 8: 3980-4000, 2017.

12. van Krieken JH, Jung A, Kirchner T, Carneiro F, Seruca R, Bosman FT, Quirke P, Fléjou JF, Plato Hansen T, de Hertogh $G$, et al: KRAS mutation testing for predicting response to anti-EGFR therapy for colorectal carcinoma: Proposal for an European quality assurance program. Virchows Arch 453: 417-431, 2008.

13. Therkildsen C, Bergmann TK, Henrichsen-Schnack T, Ladelund S and Nilbert M: The predictive value of KRAS, NRAS, BRAF, PIK3CA and PTEN for anti-EGFR treatment in metastatic colorectal cancer: A systematic review and meta-analysis. Acta Oncol 53: 852-864, 2014.

14. Zulhabri O, Rahman J, Ismail S, Isa MR and Wan Zurinah WN: Predominance of $\mathrm{G}$ to A codon 12 mutation K-ras gene in Dukes' B colorectal cancer. Singapore Med J 53: 26-31, 2012.

15. Frattini M, Saletti P, Romagnani E, Martin V, Molinari F, Ghisletta M, Camponovo A, Etienne LL, Cavalli F and Mazzucchelli L: PTEN loss of expression predicts cetuximab efficacy in metastatic colorectal cancer patients. Br J Cancer 97: 1139-1145, 2007.

16. Elbjeirami WM and Sughayer MA: KRAS mutations and subtyping in colorectal cancer in Jordanian patients. Oncol Lett 4: 705-710, 2012.

17. Guo F, Gong H, Zhao H, Chen J, Zhang Y, Zhang L, Shi X, Zhang A, Jin $\mathrm{H}$, Zhang $\mathrm{J}$ and $\mathrm{He} \mathrm{Y}$ : Mutation status and prognostic values of KRAS, NRAS, BRAF and PIK3CA in 353 Chinese colorectal cancer patients. Sci Rep 8: 6076, 2018. 
18. Tong JH, Lung RW, Sin FM, Law PP, Kang W, Chan AW, Ma BB, Mak TW, Ng SS and To KF: Characterization of rare transforming KRAS mutations in sporadic colorectal cancer. Cancer Biol Ther 15: 768-776, 2014.

19. Bamford S, Dawson E, Forbes S, Clements J, Pettett R, Dogan A, Flanagan A, Teague J, Futreal PA, Stratton MR and Wooster R: The COSMIC (Catalogue of Somatic Mutations in Cancer) database and website. Br J Cancer 91: 355-358, 2004.

20. Pai EF, Krengel U, Petsko GA, Goody RS, Kabsch W and Wittinghofer A: Refined crystal structure of the triphosphate conformation of H-ras p21 at 1.35 A resolution: Implications for the mechanism of GTP hydrolysis. EMBO J 9: 2351-2359, 1990.

21. Schindelin J, Arganda-Carreras I, Frise E, Kaynig V, Longair M, Pietzsch T, Preibisch S, Rueden C, Saalfeld S, Schmid B, et al: Fiji: An open-source platform for biological-image analysis. Nat Methods 9: 676-682, 2012.

22. Adzhubei IA, Schmidt S, Peshkin L, Ramensky VE, Gerasimova A, Bork P, Kondrashov AS and Sunyaev SR: A method and server for predicting damaging missense mutations. Nat Methods 7: 248-249, 2010

23. Kumar P, Henikoff S and Ng PC: Predicting the effects of coding non-synonymous variants on protein function using the SIFT algorithm. Nat Protoc 4: 1073-1081, 2009.

24. Reva B, Antipin Y and Sander C: Predicting the functional impact of protein mutations: Application to cancer genomics. Nucleic Acids Res 39: e118, 2011.

25. Billones JB, Carrillo MC, Organo VG, Sy JB, Clavio NA, Macalino SJ, Emnacen IA, Lee AP, Ko PK and Concepcion GP: In silico discovery and in vitro activity of inhibitors against Mycobacterium tuberculosis 7,8-diaminopelargonic acid synthase (Mtb BioA). Drug Des Devel Ther 11: 563-574, 2017.

26. Wu G, Robertson DH, Brooks CL III and Vieth M: Detailed analysis of grid-based molecular docking: A case study of CDOCKER-A CHARMm-based MD docking algorithm. J Comput Chem 24: 1549-1562, 2003.

27. Yilmaz M and Christofori G: EMT, the cytoskeleton, and cancer cell invasion. Cancer Metastasis Rev 28: 15-33, 2009.

28. Der CJ: The ras family of oncogenes. Cancer Treat Res 47: 73-119, 1989.

29. Fernández-Medarde A and Santos E: Ras in cancer and developmental diseases. Genes Cancer 2: 344-358, 2011.

30. Tojkander S, Gateva G and Lappalainen P: Actin stress fibers-assembly, dynamics and biological roles. J Cell Sci 125 1855-1864, 2012.

31. Yang SH, Yates PR, Whitmarsh AJ, Davis RJ and Sharrocks AD: The Elk-1 ETS-domain transcription factor contains a mitogen-activated protein kinase targeting motif. Mol Cell Biol 18: 710-720, 1998.

32. Cruzalegui FH, Cano E and Treisman R: ERK activation induces phosphorylation of Elk-1 at multiple S/T-P motifs to high stoichiometry. Oncogene 18: 7948-7957, 1999.

33. Hunter JC, Manandhar A, Carrasco MA, Gurbani D, Gondi S and Westover KD: Biochemical and structural analysis of common cancer-associated KRAS mutations. Mol Cancer Res 13: 1325-1335, 2015.

34. Dagogo-Jack I and Shaw AT: Tumour heterogeneity and resistance to cancer therapies. Nat Rev Clin Oncol 15: 81-94, 2018

35. Yap TA, Gerlinger M, Futreal PA, Pusztai L and Swanton C: Intratumor heterogeneity: Seeing the wood for the trees. Sci Transl Med 4: 127ps10, 2012.

36. McGranahan N and Swanton C: Biological and therapeutic impact of intratumor heterogeneity in cancer evolution. Cancer Cell 27: 15-26, 2015

37. Janakiraman M, Vakiani E, Zeng Z, Pratilas CA, Taylor BS, Chitale D, Halilovic E, Wilson M, Huberman K, Ricarte Filho JC, et al: Genomic and biological characterization of exon 4 KRAS mutations in human cancer. Cancer Res 70: 5901-5911, 2010.

38. Smith G, Bounds R, Wolf H, Steele RJ, Carey FA and Wolf CR: Activating K-Ras mutations outwith 'hotspot' codons in sporadic colorectal tumours-implications for personalised cancer medicine. Br J Cancer 102: 693-703, 2010.

39. Tejpar S, Lenz HJ, Köhne CH, Heinemann V, Ciardiello F, Beier RE, Stroh C, Duecker K and Bokemeyer C: Effect of KRAS and NRAS mutations on treatment outcomes in patients with metastatic colorectal cancer (mCRC) treated first-line with cetuximab plus FOLFOX4: New results from the OPUS study. J Clin Oncol 32: LBA444, 2017.

40. Misale S, Di Nicolantonio F, Sartore-Bianchi A, Siena S and Bardelli A: Resistance to anti-EGFR therapy in colorectal cancer: From heterogeneity to convergent evolution. Cancer Discov 4: 1269-1280, 2014
41. Murtaza BN, Bibi A, Nadeem MS, Chaudri MS and Shakoori A: Identification of a novel mutation in codon 31 of Kirstein rat sarcoma viral oncogene homologue in colon cancer: Another evidence of non-canonical mutational pathway. Pakistan J Zool 44: 1671-1676, 2012.

42. Cyniak-Magierska A, Brzeziańska E, Januszkiewicz-Caulier J, Jarzab B and Lewinski A: Prevalence of RAS point mutations in papillary thyroid carcinoma; a novel mutation at codon 31 of K-RAS. Exp Clin Endocrinol Diabetes 115: 594-599, 2007.

43. Lin SR, Tsai JH, Yang YC and Lee SC: Mutations of K-ras oncogene in human adrenal tumours in Taiwan. Br J Cancer 77: 1060-1065, 1998

44. Willumsen BM, Norris K, Papageorge AG, Hubbert NL and Lowy DR: Harvey murine sarcoma virus p21 ras protein: biological and biochemical significance of the cysteine nearest the carboxy terminus. EMBO J 3: 2581-2585, 1984.

45. Thorgeirsson UP, Turpeenniemi-Hujanen T, Williams JE, Westin EH, Heilman CA, Talmadge JE and Liotta LA: NIH/3T3 cells transfected with human tumor DNA containing activated ras oncogenes express the metastatic phenotype in nude mice. Mol Cell Biol 5: 259-262, 1985.

46. Papageorge AG, Willumsen BM, Johnsen M, Kung HF, Stacey DW, Vass WC and Lowy DR: A transforming ras gene can provide an essential function ordinarily supplied by an endogenous ras gene. Mol Cell Biol 6: 1843-1846, 1986.

47. DeFeo-Jones D, Tatchell K, Robinson LC, Sigal IS, Vass WC and Lowy DR, Scolnick EM: Mammalian and yeast ras gene products: Biological function in their heterologous systems. Science 228: 179-184, 1985

48. Cheng CM, Li H, Gasman S, Huang J, Schiff R and Chang EC: Compartmentalized Ras proteins transform NIH 3T3 cells with different efficiencies. Mol Cell Biol 31: 983-997, 2011.

49. Garrett CT and Sell S (eds): Cellular cancer markers. Humana Press, Totowa, NJ, 12, pp455-477, 1995.

50. Hynes NE and Lane HA: ERBB receptors and cancer: The complexity of targeted inhibitors. Nat Rev Cancer 5: 341-354, 2005.

51. Pirkmajer S and Chibalin AV: Serum starvation: Caveat emptor. Am J Physiol Cell Physiol 301: C272-C279, 2011

52. Chen RH, Abate $\mathrm{C}$ and Blenis J: Phosphorylation of the c-Fos transrepression domain by mitogen-activated protein kinase and $90-\mathrm{kDa}$ ribosomal S6 kinase. Proc Natl Acad Sci USA 90: 10952-10956, 1993.

53. Morton S, Davis RJ, McLaren A and Cohen P: A reinvestigation of the multisite phosphorylation of the transcription factor c-Jun. EMBO J 22: 3876-3886, 2003.

54. Reszka AA, Seger R, Diltz CD, Krebs EG and Fischer EH: Association of mitogen-activated protein kinase with the microtubule cytoskeleton. Proc Natl Acad Sci USA 92: 8881-8885, 1995.

55. Northwood IC, Gonzalez FA, Wartmann M, Raden DL and Davis RJ: Isolation and characterization of two growth factor-stimulated protein kinases that phosphorylate the epidermal growth factor receptor at threonine 669. J Biol Chem 266: 15266-15276, 1991.

56. Hong S, Kim S, Kim HY, Kang M, Jang HH and Lee WS: Targeting the PI3K signaling pathway in KRAS mutant colon cancer. Cancer Med 5: 248-255, 2016.

57. Martin TD, Samuel JC, Routh ED, Der CJ and Yeh JJ: Activation and involvement of Ral GTPases in colorectal cancer. Cancer Res 71: 206-215, 2011.

58. Chen CC, Er TK, Liu YY, Hwang JK, Barrio MJ, Rodrigo M, Garcia-Toro E and Herreros-Villanueva M: Computational analysis of KRAS mutations: Implications for different effects on the KRAS p.G12D and p.G13D mutations. PLoS One 8: e55793, 2013.

59. Jaffe AB, Aspenstrom $P$ and Hall A: Human CNK1 acts as a scaffold protein, linking Rho and Ras signal transduction pathways. Mol Cell Biol 24: 1736-1746, 2004

60. Nguyen A, Burack WR, Stock JL, Kortum R, Chaika OV, Afkarian M, Muller WJ, Murphy KM, Morrison DK, Lewis RE, et al: Kinase suppressor of Ras (KSR) is a scaffold which facilitates mitogen-activated protein kinase activation in vivo. Mol Cell Biol 22: 3035-3045, 2002.

61. Matsunaga-Udagawa R, Fujita Y, Yoshiki S, Terai K, Kamioka Y, Kiyokawa E, Yugi K, Aoki K and Matsuda M: The scaffold protein Shoc2/SUR-8 accelerates the interaction of Ras and Raf. J Biol Chem 285: 7818-7826, 2010.

62. Adzhubei I, Jordan DM and Sunyaev SR: Predicting functional effect of human missense mutations using PolyPhen-2. Curr Protoc Hum Genet, Chapter 7: Unit7.20, 2013. doi: 10.1002/0471142905.hg0720s76.

This work is licensed under a Creative Commons Attribution-NonCommercial-NoDerivatives 4.0 International (CC BY-NC-ND 4.0) License. 